\title{
OBLIGACIONES Y RESPONSABILIDAD CIVIL
}

Juan Ignacio Contardo González

Profesor de Derecho Civil

Universidad Andrés Bello

INDEPENDENCIA DE LA ACCIÓN DE PERJUiCiOS EN SEDE CONTRACTUAL. CORTE Suprema, Primera Sala (Civil), 10 de SEPTIEMBRE DE 2013, ROL 885-2013. Cita Legal Publishing: CL/JUR/ 2008/2013.

Los hechos del caso en comento (Steffen con Fundación Mi Casa) son, en resumen, los siguientes: en junio de 2011 un incendio afectó a gran parte del inmueble arrendado por la demandada. Con ocasión del siniestro, como éste ya no resultaba útil para los fines de la arrendataria (una residencia del SENAME) decidió colocar fin al contrato de arrendamiento por medio de cartas, dejando de pagar las rentas de los meses siguientes.

Frente a tal situación, la demandante dedujo, en lo principal, demanda de terminación del contrato de arrendamiento por no pago de rentas bajo el procedimiento de la ley $\mathrm{N}^{\circ} 18.101$; y en subsidio, una demanda de perjuicios en sede contractual, por los perjuicios que le acarreó el incendio del inmueble.

En primera instancia ${ }^{1}$, se rechazó la demanda de terminación del contrato de arriendo por no pago de

\footnotetext{
${ }^{1}$ Primer Juzgado de Letras de Osorno, 31 de agosto de 2012, rol 1.796-2.010.
}

rentas, porque la causal esgrimida se fundaba sólo en la falta de pago de la renta, y no en hechos distintos, como sería que un incendio afecte al inmueble y ya no sirva para los fines del arrendatario. Sin embargo, se acoge la demanda subsidiaria de perjuicios, ya que se acreditó que causó el incendio uno de los menores habitantes del inmueble, por lo que termina el fallo imputando responsabilidad a la arrendataria. La sentencia fue confirmada en segunda instancia ${ }^{2}$.

La Corte Suprema conoció el litigio por medio de la interposición de un recurso de casación en el fondo, el cual tuvo, en lo sustancial, dos capítulos. Primero, se estimó infringidas las normas reguladoras de la prueba del art. $8 \mathrm{~N}^{\circ} 7$ de la ley $\mathrm{N}^{\mathrm{O}} 18.101 \mathrm{y}$ segundo, lo que nos importa en este momento y que comentaremos, entendió infringidos los artículos 1489, 1545 y 1551 del Código Civil.

La infracción alegada a las normas del Código Civil se fundó en el hecho de que estaría vedado a los tribunales otorgar una acciones indemnizatorias directas (como la deducida en forma subsidiaria en la causa), sin previa-

${ }^{2}$ Corte de Apelaciones de Valdivia, 21 de diciembre de 2012, rol 760-2012. 
mente dar lugar o al cumplimiento o a la resolución del contrato (terminación en materia de arrendamiento). La Corte terminó por rechazar el recurso en ambos capítulos (considerando décimo cuarto y siguientes).

El principal argumento que ocupa la Corte para rechazar el recurso en el segundo capítulo, fue que la demandada incumplió una obligación de hacer: la de mantener y restituir el inmueble en estado de servir. Esta obligación sería de hacer $\mathrm{y}$, por tanto, frente a su incumplimiento, se generan los remedios alternativos concedidos en el art. 1553 del Código Civil, dentro de los cuales se encuentra la acción indemnizatoria directa $\left(\mathrm{N}^{\circ} 3\right)$.

Es decir, frente a una supuesta antinomia entre los artículos 1489, que no concedería una acción autónoma de perjuicios, y 1553, que sí la concedería, se estima que es posible aplicar la última norma por aplicación del principio de especialidad; criterio que señala la Corte Suprema, ya había sido acuñado por el máximo tribunal en sentencias anteriores. Por razones de tiempo, no comentaremos las demás infracciones alegadas, toda vez que la expuesta constituye el principal argumento que tuvo el tribunal para rechazar el recurso.

\section{LA INDEPENDENCIA DE LA ACCIÓN INDEMNIZATORIA}

Como se ha venido introduciendo, el problema al cual se aboca la Corte Suprema es la denominada "independencia" o "autonomía" de la acción indemnizatoria en sede contractual. Se trata de determinar si la acción de perjuicios puede ser demandada por sí sola o, bien, si debe acompañar una acción o de cumplimiento o de resolución de contrato. Es decir, lo que se trata de establecer es si en materia contractual se exige, por aplicación del artículo 1489 del Código Civil, una acumulación sucesiva de las acciones "principales" (cumplimiento o resolución) y la interposición de otra secundaria ("indemnizatoria") o, bien, puede deducirse la acción de daños de forma directa.

La tesis tradicional de los tribunales sostenía la "dependencia" de esta acción frente a las de cumplimiento y resolución. Los principales argumentos que se ofrecían era que el artículo 1489 así lo exigiría y, por otra parte, que frente a una acción autónoma indemnizatoria no se sabría bien qué suerte correría el contrato, ya que no se extinguiría ni por pago, ni por resolución, de tal suerte que cabría entender que el contrato se mantendría legalmente vigente, y subsistirían las acciones de cumplimiento y resolución.

Esta tesis, que estaba ya bien asentada en los tribunales superiores, comienza a cambiar con la sentencia de la Corte Suprema conocida como Opazo Lamana y otros con Inmunomédica Laboratorio Limitada ${ }^{3}$, en que se le reconoce independencia a la acción indemnizatoria, frente a la de cumplimiento. En lo sucesivo, otras sentencias de los tribunales superiores fueron por la misma senda ${ }^{4}$.

${ }^{3}$ Corte Suprema, 7 de diciembre de 2010, rol 3341-2009.

${ }^{4}$ Así, por ejemplo, puede citarse Faúndez con Compañía de Telecomunicaciones de Chile 
En el plano doctrinal, antes de la sentencia recaída en Opazo, ya se habían pronunciado algunos autores. Especial influencia tuvo un artículo publicado en esta revista, de autoría de la profesora Patricia López, que incluso fue citado en el fallo antes mencionado ${ }^{5}$.

Con todo, parece importante señalar que de todas formas la doctrina le reconoció independencia a la acción de perjuicios cuando la obligación era de hacer, precisamente, por lo dispuesto en el No 3 del artículo $1553^{6}$. La dificultad se presentaba en las obligaciones de dar, en que a falta de norma expresa, la autonomía de la acción indemnizatoria se discutía.

S.A., Corte de Apelaciones de Concepción, 29 de marzo de 2011, rol 1258-2010, N Legal Publishing: 48526; Asociación de Productores de Huevos de Chile con Banco de Chile, Corte Suprema, 31 de agosto de 2011, rol 1061-2010, base de datos www.vlex.com, $\mathrm{N}^{\circ} 333763730$; Reyes Valdivia con Escuela de Tripulantes y Portuaria, Corte Suprema, 10 de julio de 2012, rol 4328-2012, N Legal Publishing: CL/ JUR/1308/2012; Zorín S.A. con Compañía Siderúrgica Huachipato S.A, Corte Suprema, 31 de octubre de 2012, rol 3325-12, $\mathrm{N}^{\circ}$ Legal Publishing: CL/JUR/2412/2012; Sociedad Ingeniería y Construcción del Sur S.A. Con fisco de Chile, Corte Suprema, 20 de noviembre de 2012, rol 3538-2012, N Legal Publishing: CL/JUR/2468/2012; Ampuero con Castillo, Corte Suprema, 28 de enero de 2013, , rol 58982012 N $^{\circ}$ Legal Publishing: CL/JUR/174/2013.

${ }^{5}$ LÓPEZ (2010) passim. En este artículo se da cuenta detallada de los argumentos doctrinales como jurisprudenciales para rechazar la denominada "autonomía" y se ofrecen algunos a su favor, los que son recogidos en parte por Opazo. Por lo tanto, nos remitimos al artículo de la profesora Patricia López en su totalidad.

${ }^{6}$ Así lo reconocen, por ejemplo, algunos manuales de consulta frecuente: MEZA (2007), pp. 120-121; Ramos (2008), pp. 241-242. También parte de nuestra doctrina más moderna: PIZARRO (2010), p. 310; LÓPEZ (2010), p.. 83.
De vuelta al caso en comento (Steffen con Fundación Mi Casa), parece ser que esta sentencia va en la misma línea que ya trazaba Opazo, esto es, de otorgar independencia a la acción de daños. Aun cuando esto no resulta del todo novedoso, pues le dio aplicación directa al art. 15537, según lo comentado.

\section{EL SUPUESTO CARÁCTER ESPECIAL} DEL ARTÍCULO 1553 FRENTE Al ARTíCUlo 1489 DEL Código CIVIL

Sin embargo, a nuestro entender, el criterio de especialidad seguido por la Corte Suprema para aplicar el art. 1553 en desmedro del artículo 1489, no es del todo correcto. Hay argumentos para otorgar independencia sustantiva a la acción indemnizatoria (más allá de los procesales), sin acudir al criterio de especialidad.

En primer lugar, no puede desconocerse que los contratos bilaterales contengan obligaciones de hacer. Por tanto, descartar el artículo 1489 por ser más general que el artículo 1553 no nos parece que sea un argumento satisfactorio, desde el momento que incluye todos los casos de contratos bilaterales, sean sus obligaciones de hacer, no hacer o de dar. Debe tenerse presente que la aplicación del principio de especialidad tiene lugar cuando dos normas imputan a un mismo caso soluciones que son incompatibles entre sí, de tal manera que el intérprete da preferencia "a la norma específica que está en conflicto con una cuyo campo

\footnotetext{
${ }^{7}$ Para un recuento jurisprudencial, véase LÓPEZ (2010), p. 76.
} 
de referencia sea más general"8. Este campo de referencia aparentemente más general del art. 1489 nos da la impresión que, incluso, puede llegar a la interpretación contraria. De hecho, la configuración normativa del artículo 1553 parece estar pensada, más bien, para contratos "unilaterales de hacer", por la sencilla razón que no determina qué suerte corre el contrato incumplido para la otra parte. Es decir, regula los derechos del acreedor frente al obligaciones de hacer, pero no es autónomo para determinar qué otros derechos nacen en el marco de una relación obligacional bilateral. Estos efectos están disciplinados por los artículos 1489, 1550 en materia de riesgos (aunque limitado a las entregas de especies y cuerpos ciertos) y 1552 para los incumplimientos recíprocos. Si estamos en lo correcto, el argumento de aplicación especial del art. 1553 frente al art. 1489 en realidad no es del todo satisfactorio, puesto que no encontramos una inconsistencia suficiente entre ambas normas para dar aplicación preferente a una sobre la otra. Por tanto, creemos que el problema de fondo tiene relación con hacer coincidir los derechos del art. 1553 con el artículo 1489, y no al revés, como es frecuente que se analice la cuestión.

Asimismo, debe recordarse que por razones sistemáticas se incluyó el art. 1489 en el título de las obligaciones condicionales y no en el título de los efectos de las obligaciones, por entender que la resolución por incumplimiento era una condición, opinión que hoy día se encuentra en franca retirada. Si se lee la norma como una

${ }^{8}$ Nino (2012), pp. 272-275.

disposición que regula los efectos del incumplimiento de los contratos bilaterales, entonces el problema interpretativo denunciado se hace más patente, y conviene hacer una relectura de las normas de incumplimiento que permita dar una interpretación más armónica a todo el texto, sin excluir el art. 1489 por principio de especialidad.

EL CENTRO DEL PROBLEMA:

LA INDEMNIZACIÓN DE DAÑOS DESLIGADA DEL VALOR DE PRESTACIÓN

En segundo lugar, parece que no está bien delimitado el concepto de indemnización de perjuicios que, como se verá, es de importancia para determinar la "independencia" de la acción.

Si se lee cualquiera de nuestros más célebres manuales y tratados de obligaciones en la parte que abordan la responsabilidad contractual, se observa que la doctrina en general asimila el concepto de indemnización de perjuicios al cumplimiento por equivalencia ${ }^{9}$. El cumplimiento por equivalencia es, precisamente, el sustituto (equivalente) dinerario de la prestación en naturaleza.

Por lo tanto, según este concepto de indemnización el problema de la "independencia" de la acción debiera desplazarse a otro: si es posible demandar al arbitrio del acreedor el cumplimiento en naturaleza o por equivalencia. Como se señaló, la respuesta de la doctrina y jurisprudencia es relativamente uniforme: se podría sólo en las obligaciones

${ }^{9}$ Puede citarse como ejemplo: GATiCA (1959), p. 11 y ss; FueYo (2004), pp. 342-343; MEZA (2007), p. 119; AbeLiuk (2008), pp. 810-811. 
de hacer (art. $1553 \mathrm{~N}^{0} 3$ ) y en algunos casos en las de no hacer (art. 1555). A falta de norma, sería discutible en las obligaciones de dar. Sin embargo, hay bastantes buenas razones para su aceptación especialmente por la regulación procesal del juicio ejecutivo en las obligaciones de dar objetos no específicos, en que se permite su avaluación.

Así, sería posible sostener que el 1553 , en sus tres numerandos, contiene distintas formas de cumplimiento. El $\mathrm{N}^{\circ} 1$, es una pretensión de cumplimiento en naturaleza: "Que se apremie al deudor para la ejecución del hecho convenido".

Pero, a nuestro entender, los dos últimos comprenden formas de cumplimiento por equivalencia. De esta manera, el $\mathrm{N}^{\mathrm{o}} 2$ que establece:

"Que se le autorice a él mismo para hacerlo ejecutar por un tercero a expensas del deudor",

contiene una forma de cumplimiento que en apariencia es en naturaleza: la ejecución de la obra por un tercero (estamos en el caso de obligaciones de hacer fungibles, por ello las puede cumplir el tercero). Con todo, la ejecución recae sobre el patrimonio del deudor para dar pago al tercero, según el régimen de las obligaciones de dar. Esto implica, necesariamente, que se transforma la ejecución en un cumplimiento por equivalencia, según el régimen ejecutivo de las obligaciones de dar (art. 541 del CPC).

$\mathrm{Y}$ el tercer numerando:

"Que el deudor le indemnice de los perjuicios resultantes de la infracción del contrato", parece ser coincidente también con una pretensión de cumplimiento por equivalente. Esta conclusión puede obtenerse a partir del encabezado del art. 1553. Si se observa con detención la norma, esta "indemnización" es complementaria con la moratoria permitida por el mismo artículo ("junto con la indemnización de la mora"). De esta manera habrían dos "indemnizaciones" que podrían ser demandadas conjuntamente: la "moratoria" del encabezado y la permitida por el $\mathrm{N}^{\mathrm{o}} 3$, que sería la compensatoria (en el sentido de que se le atribuye por nuestra doctrina más clásica, es decir, la de un cumplimiento por equivalente).

Si se lee de esta manera la norma, entonces, no habría ninguna contradicción entre el art. 1489 y el 1553. A nuestro entender, la pretensión de cumplimiento del art. 1489 comprende cualquiera de los tres derechos conferidos para el acreedor de obligaciones de hacer: ya se trate de un cumplimiento en naturaleza, ya se trate de un cumplimiento por equivalencia. Desde este punto de vista, no hay un ejercicio "autónomo" de la indemnización de perjuicios, porque está subsumida en la pretensión de cumplimiento. Ambas normas serían coincidentes y complementarias. En esto, si se sigue nuestra opinión, la sentencia de la Corte no sería adecuada.

\section{CumPlimiento POR EQUIVALENTE E INDEMNIZACIÓN DE DAÑOS: UNA DISTINCIÓN QUE PODRÍA OTRORGAR MÁS CLARIDAD}

Si se ha estado en lo correcto hasta el momento, surge una nueva inte- 
rrogante, y que el caso lo coloca de manifiesto. Al colocar mirada en los daños reclamados en el caso, será posible observar que se demandó los perjuicios sufridos con ocasión del incendio, lo que tiene relación con básicamente el costo de reparación del inmueble (por adherencia) al estado en que se encontraba al momento de ser entregado y las rentas que dejó de percibir hasta el termino del contrato (considerandos vigésimo primero y vigésimo segundo, fallo de primera instancia). ¿Se trata de un cumplimiento por equivalente?

Adelantamos que la respuesta no es fácil, pues incorpora algunas sutilezas que al final del día hacen una gran diferencia.

La idea de un cumplimiento por equivalente surge de la necesidad de reemplazar el objeto específico (o cuerpo cierto) perdido por culpa del deudor o durante la mora del mismo. Como es un cuerpo cierto, no habría como reemplazarlo, sólo con dinero. Como establece el Código, en estos casos la obligación no se extingue, subsiste, pero varía de objeto (art. 1672). Sólo se extinguirá la obligación (y consecuentemente la responsabilidad del deudor), cuando la pérdida es fortuita (1670). Por tanto, cuando perece el objeto específico, "el deudor es obligado al precio de la cosa y a indemnizar al acreedor", cuando la pérdida se debe a la culpa del deudor o después de su constitución en mora (en el lenguaje del Código).

Esta noción de reemplazo de una especie o cuerpo cierto por dinero está relacionada, con la pérdida del objeto: como es específico, no habría como reemplazarlo, ya que no hay otro igual. Hoy día, como la regla general en el tráfico (no en el Código) es el género, la idea de reemplazo por otro equivalente parece tener bastante lógica ${ }^{10}$. En efecto, el género por regla general no perece (art. 1510). Cabe hacer presente que en una sociedad en que el género prevalece, el cumplimiento por equivalente puede hacerse ya en dinero, ya en otras cosas que equivalgan al objeto prometido ${ }^{11}$. Incluso, la posibilidad de reparación de objetos (o también de hechos), que es un remedio reconocido con generalidad en el Derecho Contractual Uniforme. Sobre esto último volveremos un poco más adelante.

Luego, la idea de un cumplimiento por equivalente (sólo en dinero) como daño, se diluye. Más todavía cuando el costo de sustitución supone una diferencia monetaria entre lo adquirido en origen y lo que sustituye, que puede ser mayor o menor, aun de mejor calidad (a menor precio).

De aquí, entonces, que pueda reconducirse la problemática a una cuestión ya no de cumplimiento (por equivalencia), sino de perjuicios en un sentido muy estricto: la pérdida patrimonial que apareja el reemplazo ${ }^{12}$.

${ }^{10}$ Sobre el particular, véase ALCALDE (2013), p. $99 \mathrm{y}$ ss.

${ }^{11}$ Jaime Alcalde distingue tres modalidades de sustitución: económica, jurídica y negocial. El cumplimiento por equivalente comportaría una sustitución económica: Alcalde (2013), p. 109.

${ }^{12}$ Se trata de una visión un tanto restrictiva de lo que debiera considerarse como daño contractual, cuyas bases dogmáticas se pueden encontrar en la distinción hecha por el artículo 1672 entre la aestimatio rei $\mathrm{e}$ id quod interest. Esta distinción fue propuesta por Jorge Baraona hacia 
Éste es el sentido, por ejemplo, que se le da a la noción de daño o perjuicio contractual en los artículos 75 y 76 de la Convención de Viena sobre Compraventa Internacional de Mercaderías.

Vistas las cosas desde esta óptica, es posible concebir una noción de perjuicio que es un tanto diferente al cumplimiento por equivalente. Piénsese ahora sobre la noción de lucro cesante, ¿tiene alguna relación con el cumplimiento por equivalente?, ¿̇y qué sucede con el daño moral?

De esta suerte, es posible configurar una noción de perjuicio en sentido estricto: aquél que no está relacionada con el valor de prestación, sino en el daño que sufre el acreedor distinto en la pérdida de la prestación ${ }^{13}$. Y esto sí está reconocido en el Código.

Si se vuelve la mirada al art. 1672, recordemos que la pérdida "culpable" del cuerpo cierto por parte del deudor, éste es obligado al "precio de la cosa y a indemnizar al acreedor". El valor de prestación ya está incorporado en lo que se denomina "precio", pero el daño sufrido por la pérdida no está en él. Se trata de algo distinto: la pérdida que le ha significado al acreedor la posibilidad de no disponer de la cosa, por "culpa" del deudor. Y esto no es sino la indemnización de perjuicios $^{14}$.

De vuelta al caso, recordemos que el arrendatario debió restituir la cosa en el mismo estado en que fue reci-

1997 con relativa aceptación en la doctrina. Cfr. BaRAona (1997), p. 159 y ss.; Bustamante (2005), pp. 105-126; CORRAL (2010), p. 141 y ss.; Peñailillo (2009), pp. 334-338.

${ }^{13}$ BaraOna (1997), pp. 159-162.

${ }^{14}$ Op. cit., pp. 156-159. bida, descontado el uso natural de la misma. Pero tratándose de un incendio, como establecieron los tribunales, en especial de instancia, lo verdaderamente incumplido por el deudor fue la entrega de la cosa en el estado debido. Por ello decretaron, como indemnización, el valor de los costos de reparación del inmueble y la renta que dejó de percibir hasta el término del contrato.

Entonces, ¿ cómo calificar la pérdida del acreedor/arrendador? (en definitiva recibió, por vía de restitución, un inmueble quemado).

Es probable que las disquisiciones teóricas antes enunciadas puedan oscurecer la cuestión. Pero nos da la impresión que no es discutible que si el incendio fue causado por uno de los habitantes del inmueble, debiera el arrendatario responder (en el sentido amplio de la acepción). El problema es a qué título: si de cumplimiento o de indemnización.

La importancia de la discusión radica en los requisitos que deben exigirse a ambos remedios. Tal como ha hecho presente parte de la doctrina actual, la pretensión de cumplimiento requiere de requisitos más básicos que la indemnización. Requeriría sólo del incumplimiento, y no la mora del deudor, ni su culpa en la inejecución, tampoco la relación de causalidad y menos el daño. Por el contrario, la indemnización, todos los enunciados.

Ahora, si vemos bien, lo que hay detrás de la reclamación del actor no es sólo la restitución material del suelo sino la restitución de la cosa en estado de servir como fue entregada (art. 1947). De esta manera, creemos 
que hay detrás de la reclamación del actor una pretensión de cumplimiento: la restitución de la cosa en estado de servir a través de la reparación del mismo. Así las cosas, más que una indemnización de perjuicios nos encontramos con una pretensión de cumplimiento a través de una forma un tanto particular: la reparación del objeto. Sin embargo, ella no resulta extravagante en el nuevo Derecho de la Obligaciones: la reparación es un recocida como un remedio alternativo al cumplimiento. Y, para aquellos Códigos que no la tengan establecida expresamente dentro de su catálogo de remedios (como el chileno), Antonio Manuel Morales Moreno opina que debe encuadrarse dentro de las pretensiones de cumplimiento ${ }^{15}$.

Y esta pretensión de cumplimiento va acompañada de otra: la reclamación de las rentas que quedaban por pagar. ¿No se está más que pidiendo aquello que dejó de percibir (lucro cesante), el cumplimiento de la obligación principal del arrendatario, esto es, el pago de la renta?

Si estamos en lo correcto, en realidad lo que estaba encubierto a través de una reclamación por daños es una pretensión de cumplimiento. La que reiteramos tiene requisitos de operatividad más básicos que la indemnización de perjuicios. Y lo que importa, para los efectos de la "autonomía" de la indemnización, es que no era necesario buscar interpretaciones que permitieran darle este carácter. Como se trata de una pretensión de cumplimiento, luego, bastaba con aplicar los arts. 1489 y 1553 en el sentido propuesto.

${ }^{15}$ Morales (2006), p. 198.
A nuestro entender, buena parte del problema de la "independencia" o "autonomía" de la acción indemnizatoria pasa, en primer lugar, por realizar un estudio comprehensivo de la noción de daño contractual. Muchas veces bajo una "indemnización”, en realidad encontramos otras cosas: una pretensión de cumplimiento (como sucedió en el caso) o una acción restitutoria derivada de la operatividad de la resolución (art. 1487). La falta de claridad sobre el concepto de daño produce algunas confusiones que han requerido realizar esfuerzos interpretativos para dar cabida a su "autonomía", cuando ello no siempre es necesario (como la de la supuesta antinomia entre los arts. 1553 y 1489). Lo anterior no significa que la indemnización de perjuicios no se justifique en algunos casos de ser ejercida de manera "autónoma". Pero, para ello, creemos que debe analizarse primero si la pretensión tiene un real contenido indemnizatorio. Se trata de observar con algo más de detención cuál es el interés preciso del acreedor y encausarlo dentro del catálogo de remedios disponibles.

\section{BibliograFÍA}

Abeliuk Manasevich, René (2008). Las obligaciones. Santiago: Editorial Jurídica de Chile.

Alcalde Silva, Jaime (2013). Incumplimiento y reemplazo. Bases para una teoría general de la operación de reemplazo en el derecho español. (tesis para optar al grado de doctor en Derecho). Valencia: inédita. Disponible en http://roderic.uv.es/bitstream/ 
handle/10550/28899/Tesis $\% 20$ doctoral $\% 20$ Jaime $\% 20$ Alcalde $\% 20$ Silva.pdf? sequence $=1$. $[$ Fecha de consulta: 9 de abril de 2014].

Baraona GonZÁlez, Jorge (1997). "Responsabilidad contractual y factores de imputación de daños: apuntes para una relectura en clave objetiva”. Revista Chilena de Derecho, vol. 24, $\mathrm{N}^{\circ}$ 1. Santiago.

Bustamante Salazar, Luis (2005). "Autonomía del equivalente pecuniario o su integración dentro de la indemnización de daños y perjuicios", en Juan Andrés Varas Braun y Susan Turner Saelzer (coords), Estudios de Derecho Civil. Santiago: LexisNexis.

Corral Talciani, Hernán (2010). "La extensión del resarcimiento de los daños contractuales: las reglas de la causalidad y de la previsibilidad", en Hernán Corral Talciani, Contratos y daños por incumplimiento. Santiago: Abeledo Perrot-Legal Publishing.

Fueyo Laneri, Fernando (2004). Cumplimiento e incumplimiento de las obligaciones. Santiago: Editorial Jurídica de Chile.

Gatica Pacheco, Sergio (1959). Aspectos de la indemnización de perjuicios por incumplimiento de contrato. Santiago: Editorial Jurídica de Chile.
López Díaz, Patricia Verónica (2010). "La indemnización compensatoria por incumplimiento de los contratos bilaterales como remedio autónomo en el Derecho Civil Chileno". Revista Chilena de Derecho Privado, $\mathrm{N}^{\circ}$ 15. Santiago.

Meza Barros, Ramón (2007) Manual de Derecho Civil. De las obligaciones. Santiago: Editorial Jurídica de Chile.

Morales Moreno, Antonio Manuel (2006). "El derecho a la reparación o sustitución de la cosa no conforme y la naturaleza de la obligación del vendedor", en Antonio Manuel Morales Moreno. La modernización del derecho de obligaciones. Madrid: Civitas.

Nino, Carlos (2012). Introducción al análisis del derecho. Buenos Aires: Astrea.

Peñailillo Arévalo, Daniel (2009). "Responsabilidad contractual objetiva", en Carlos Pizarro Wilson (coord.), Estudios de Derecho Civil IV. Santiago: Legal Publishing, 2009.

Pizarro Wilson, Carlos (2010). "La responsabilidad contractual en el derecho chileno: función y autonomía", en Carlos Pizarro WiLson y Álvaro VIDAL Olivares. Incumplimiento contractual, resolución e indemnización de daños. Bogota: Editorial Universidad del Rosario. 